\title{
Caustics, Orthotomics, and Reflecting Curve with Source at an Infinity
}

\author{
Aleksandr Dvoretskii ${ }^{[0000-0002-1152-768 X]}$ \\ The Crimean Federal University named after V.I. Vernadsky, Simferopol, Russia \\ erces_crimea@mail.ru
}

\begin{abstract}
The properties of the first reflection of curves can be investigated by their connections. It is giving us the possibility to construct new reflecting curves and reflecting systems with the demand properties. The studied reflective properties of plane curves as sections of reflecting surfaces are preserved if these sections are generators of surfaces of revolution and rotative surfaces (the normal to the section at a given point coincides with the normal to the surface at the same point) and the source of the incident rays is in the same plane section.

Caustics, orthotomics, and reflective curves with a source at an infinity are considered. For a given orthotomics, there is a one-parameter set of reflective curves with a source at an infinity. To select one reflecting curve from this set it is necessary to put straight line, perpendicular to the direction of the incident rays. The shaping of a reflecting curve and its caustics according to its given orthotomics, as well as the shaping of orthotomics according to a given reflective curve with a source at an infinity, is studied, and algorithms for these shaping are proposed. Equations are written for all studied curves.

Caustics, orthotomics, and reflective curves with a source at infinity are a promising means of using the methods of geometric optics for modeling natural objects in ergo design.
\end{abstract}

Keywords: Orthotomic, Caustic, Shaping of a Reflecting Curve, a Source at an Infinity

\section{Statement of the problem}

When the source is located in the plane of the reflecting curve, the envelope of the reflected rays can be found, which is called caustic. Singularities of caustics, systems of rays and fronts were studied by Huygens even before the emergence of mathematical analysis (1654). In the works of Cayley (1878), studies of the singularities of wave fronts and caustics made significant progress [1]. Caustics for a circle with a light source at point $\mathrm{L}$ are shown in Figure 1 and described in [2].

Copyright (C) 2020 for this paper by its authors. Use permitted under Creative Commons License Attribution 4.0 International (CC BY 4.0). 


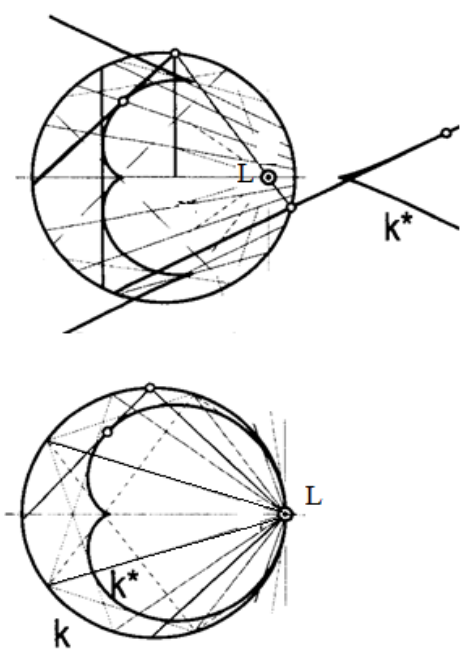

Fig. 1. Caustic for a circle

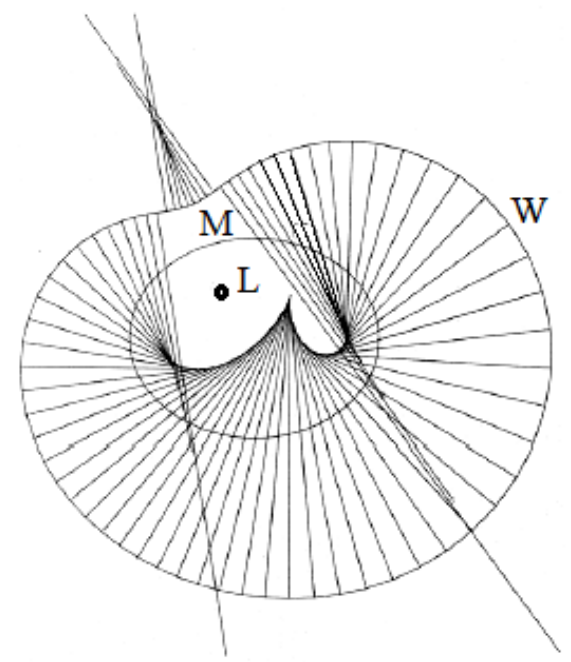

Fig. 2. Caustic and orthotomic W of an ellipse $\mathrm{M}$ with a point source $\mathrm{L}$ located out of focus.

The envelope of the normals of a curve is known as its evolute (and can also be thought of as the set of canters of curvature of the curve). Thus, focusing occurs on the evolute of $\mathrm{W}$, called the orthotomic of $\mathrm{M}$ relative to $\mathrm{L}$. Because of this optical connection, the evolute is also known as the focal set of $\mathrm{W}$, or indeed the caustic, but we reserve this term for a caustic by reflection. The orthotomic was introduced into the study of caustics by Quetelet in 1826. Caustics themselves appear to go back as far as Tschirnhaus (1682); a detailed study of special cases was carried out by Cayley [9] in 1856.

In the works of Bruce, Gibling, Gibson [3,4], caustics and orthotomics of an ellipse are considered for a point source located out of focus. Figure 2 shows the reflection from an ellipse $\mathrm{M}$ with a source $\mathrm{L}$, located inside the ellipse.

The objective of this article is to study the relationship between caustics, orthotomics and a reflective curve with a source at an infinity.

The elements included in this apparatus are: catacaustics $\mathbf{n}$ - a curve enveloping a one-parameter set of reflected rays and at the same time being an evolute of an orthotomic $\mathbf{o}$; podder curve $\mathbf{p}$, which is the locus of points equidistant from orthotomics and the corresponding point $\mathrm{L}$ on line $\mathbf{1}$.

For a given orthotomics, there is a one-parameter set of reflective curves with a source at an infinity. It is possible to select one reflecting curve from this set by specifying a straight line $\mathbf{1}$, perpendicular to the direction of the incident rays $S$ (see Fig. 2). The theory of quasi-focal lines for reflections in three-dimensional space is described in [6]. 


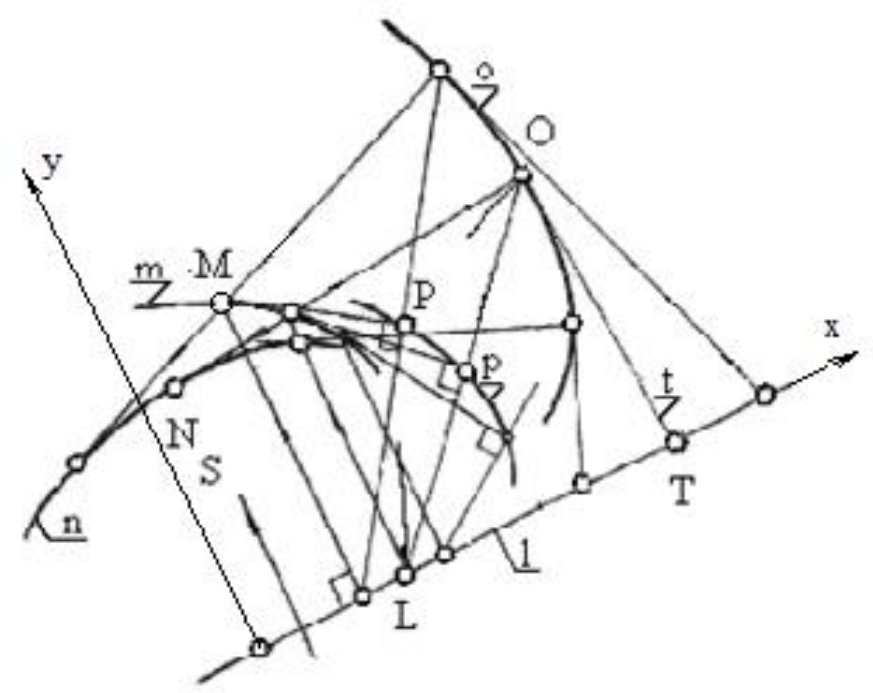

Fig. 3. The reflecting apparatus with a source at an infinity

\section{Formation of the reflecting curve and its caustic} according to its given orthotomic with a source at an infinity

It is proposed to construct a reflecting curve according to its given orthotomic according to the following algorithm:

1. A point $0(\mathrm{x}, \mathrm{y})$ is chosen on the orthotomic $\mathbf{o}$ and the tangent $\mathbf{t}$ is constructed in this point.

2. From the point $\mathrm{T}$ as a center, which is the point of intersection of tangent $\mathbf{t}$ and straight line 1, a circle with radius $\mathrm{TN}$ cuts a point $\mathrm{L}$ on straight line 1 .

3. The point of intersection of the normal to the orthotomic at point $\mathrm{O}$ with the incident ray, passing through point $\mathrm{L}$ is the point of the reflecting curve $\mathrm{M}(\mathrm{x}, \mathrm{y})$.

Let

$$
y=f(x)
$$

is the orthotomics equation o, for which we find the reflecting curve $\mathrm{m}$, and as a straight line that distinguishes one reflecting curve, we take the $\mathrm{x}$ axis (see Fig. 3). The tangent to the curve $\mathrm{o}$ at the point $\mathrm{O}(\mathrm{x}, \mathrm{y})$ is described by the equation

$$
Y-y=f^{\prime}(x)(X-x),
$$

where $\mathrm{x}$ and $\mathrm{y}$ are current coordinates.

Coordinates of point $\mathrm{T}$, cut off by tangent (2) on the $\mathrm{x}$-axis are

$$
X_{T}=\frac{f^{\prime}(x) \cdot x-y}{f^{\prime}(x)} ; \quad Y_{T}=0 .
$$


4 A. Dvoretskii.

The distance from point $\mathrm{T}$ to point $\mathrm{N}$ will be

and with (3) is

$$
d=\left(X_{T}-x\right)^{2}+\left(Y_{T}-y\right)
$$

$$
d=y \cdot \sqrt{1+\frac{1}{f^{\prime}(x)^{2}}} .
$$

Then the equation of the incident ray perpendicular to the $\mathrm{x}$-axis at point $\mathrm{L}$ will be:

$$
X_{L}=X_{T}-d=\frac{f^{\prime}(x) \cdot x-\left(1+\sqrt{1+f^{\prime}(x)^{2}}\right) \cdot y}{f^{\prime}(x)}=X_{M} .
$$

The equation of orthotomics normal is

$$
Y-y=-\frac{1}{f^{\prime}(x)}(X-x) \text {. }
$$

The coordinates of the point $\mathrm{M}$ of the reflecting curve are determined by the intersection of the normal to the orthotomics (7) and the incident ray (6) passing through point $\mathrm{L}$.

Taking into account (1) and (3) we get

$$
\begin{gathered}
X_{M}=\frac{f^{\prime}(x) \cdot x-\left(1+\sqrt{1+f^{\prime}(x)^{2}}\right) \cdot y}{f^{\prime}(x)}, \\
Y_{M}=\frac{\left(f^{\prime}(x)^{2}-1-\sqrt{1+f^{\prime}(x)^{2}}\right) \cdot y}{f^{\prime}(x)^{2}} .
\end{gathered}
$$

This is the parametric equation of the reflective curve.

As an example, consider finding a reflective curve for orthotomic in the form of a circle centered on the $\mathrm{y}$-axis and a straight line 1 coinciding with the $\mathrm{x}$-axis. Let

$$
\left\{\begin{array}{l}
x=R \cdot \cos t \\
y=R \cdot \sin t+a
\end{array} .\right.
$$

is the parametric equation the circle $\mathbf{o}$

Derivative of the equation (10) will be

$$
y^{\prime}(x)=\frac{y^{\prime}(t)}{x^{\prime}(t)}=-c t g t .
$$




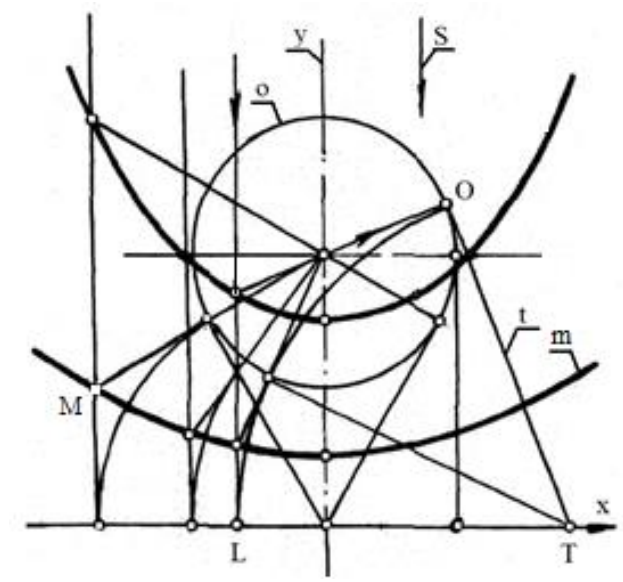

Fig. 4. Reflecting curve for the orthothomic in the form of circle $\mathbf{o}$ is parabola $\mathbf{~} \mathbf{m}$.

Using formulas (10) and (11), we obtain the coordinates of the current point of the reflecting curve $\mathbf{m}$, depending on the parameter $\mathbf{t}$. After excluding the parameter $t$, the equation takes the form:

$$
x^{2}=2(a-R) \cdot y+R^{2}-a^{2} .
$$

Equation (12) shows that the reflecting curve for circular orthotomic is a parabola (see Fig. 4).

Table 1 shows the reflecting curves for orthotomics in the form of an ellipse, parabola, and cycloid, and their caustics. Figure 5 shows the result of the formation of four curves by the reflections with a source at an infinity. The calculations were performed in the MathCad program.

Table 1. Connection of orthothomic and caustic

\begin{tabular}{|l|l|l|}
\hline $\begin{array}{c}\text { Ellipse } \\
x=a \cdot \cos t\end{array}$ & Drawing \\
\hline$y=b \cdot \sin t$
\end{tabular}


6 A. Dvoretskii.
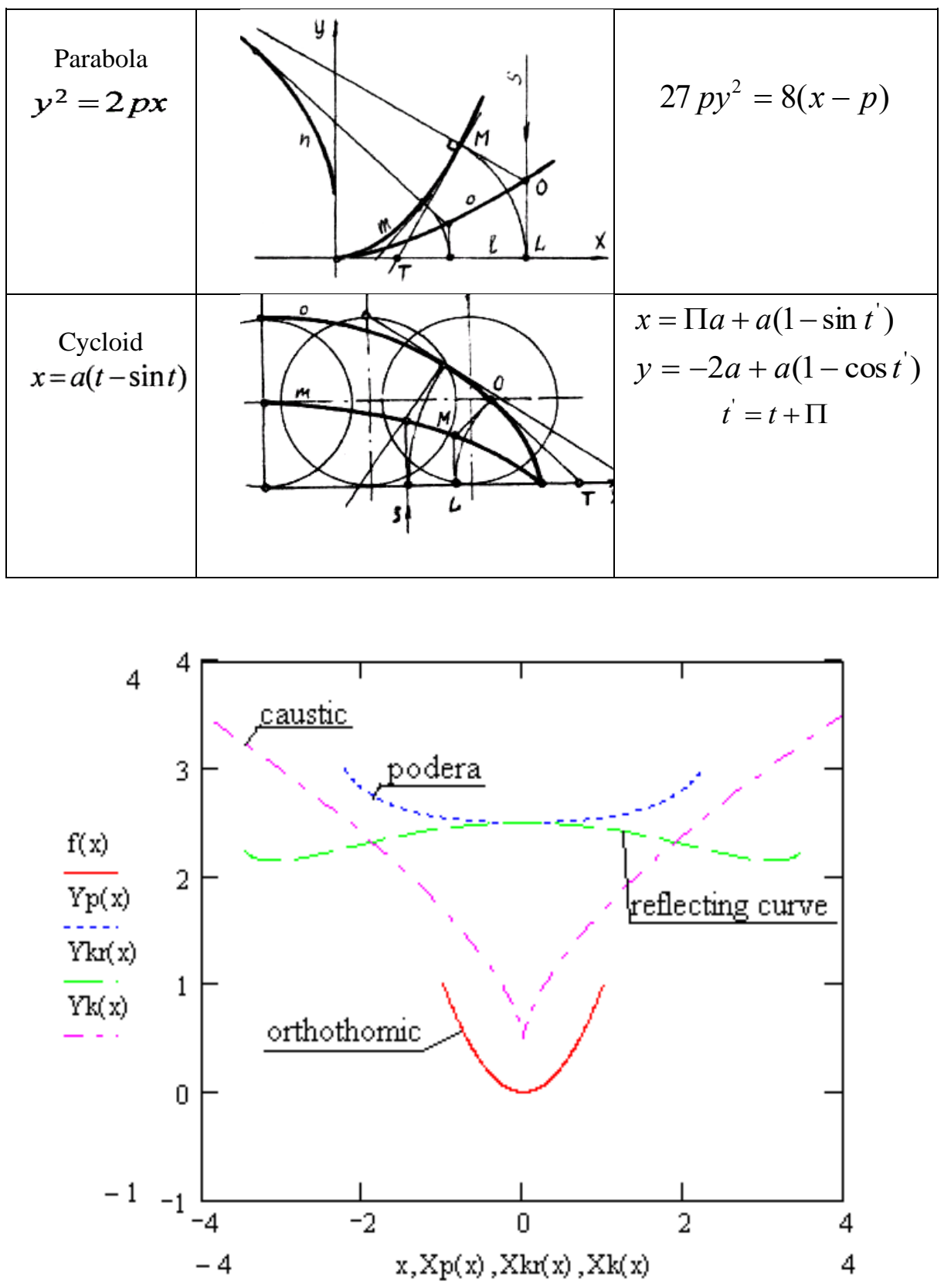

Fig. 5. Reflecting curves on the given orthothomic as a parabola. 
Table 2. Reflecting curves on the given orthothomic as a parabola

\begin{tabular}{|c|c|c|c|}
\hline Reflecting curve & Podera & Othothomic & Caustic \\
\hline$x_{k r}=x-(b-y) \cdot \operatorname{tg} \chi$ & $x_{p}=\frac{4 \cdot p \cdot x}{4 \cdot p}-$ & & $x_{k}=\frac{-x^{3}}{p^{2}}$ \\
$y_{k r}=\frac{x^{3}+p \cdot \operatorname{tg} \chi}{2 \cdot x \cdot p}$. & $-\frac{\left(2 \cdot b \cdot p-x^{2}\right) \cdot \operatorname{tg} x}{4 \cdot p}$ & $y=\frac{x^{2}}{2 \cdot p}$ & $y_{k}=\frac{2 \cdot p^{2}+3 \cdot x^{2}}{2 \cdot p}$ \\
$\cdot\left(2 \cdot b \cdot p-x^{2}\right)$ & $y_{p}=\frac{2 \cdot b \cdot p+x^{2}}{4 \cdot p}$ & & \\
& & & \\
\hline
\end{tabular}

\section{Shaping of orthotomics according to a given reflective curve with a source at an infinity}

Since an orthotomic determines the reflective properties of the curve, it is advisable to consider the shaping of orthotomics on a given reflective curve with a source at an infinity.

Statement. For a given reflecting curve $\mathrm{m} y=\mathrm{f}(\mathrm{x})$, orthotomics is one of two envelopes of a set of circles of variable radius centered on the reflecting curve. Another envelope is a straight line 1 , which defines a beam of parallel incident rays and is perpendicular to these rays (Fig. 6).

Finding one of the envelopes, if the other is a straight line, is proposed using the following algorithm:

1. The point $M(x, y)$ on the reflecting curve $m, y=f(x, y)$, is selected.

2. Construct a tangent $t$ to the reflecting curve at the point $M(x, y)$.

3. Find a point $\mathrm{A}$ on line $\mathbf{1}$, simultaneously belonging to the incident ray passing through the point $\mathrm{M}(\mathrm{x}, \mathrm{y})$.

4. Point $\mathrm{O}$ (Xo.Yo) of orthotomic is constructed as a point symmetric to point A relative to the tangent $\mathbf{t}$ at the point $\mathrm{M}(\mathrm{x}, \mathrm{y})$.

Let a reflecting curve in the form of a circle be given (Fig. 6)

$$
(y-a)^{2}+x^{2}=R^{2} \text {. }
$$

The equation of the tangent $\mathrm{t}$ to the reflecting curve at the point $\mathrm{M}(\mathrm{x}, \mathrm{y})$ is

$$
Y-y=f^{\prime}(x)(X-x)
$$

From equation (13)

$$
y-a=\sqrt{R^{2}-x^{2}}
$$


8 A. Dvoretskii.

from here

$$
f^{\prime}(x)=\frac{d y}{d x}=-\frac{x}{\sqrt{R^{2}-x^{2}}},
$$

and the tangent equation takes the form

$$
Y=-\frac{x}{\sqrt{R^{2}-x^{2}}} \cdot X+y+\frac{x^{2}}{\sqrt{R^{2}-x^{2}}} .
$$

Equation of the perpendicular dropped from the point $\mathrm{A}(\mathrm{x}, 0)$ to the tangent $\mathbf{t}$

$$
Y=\frac{\sqrt{R^{2}-x^{2}}}{x}(X-x) \text {. }
$$

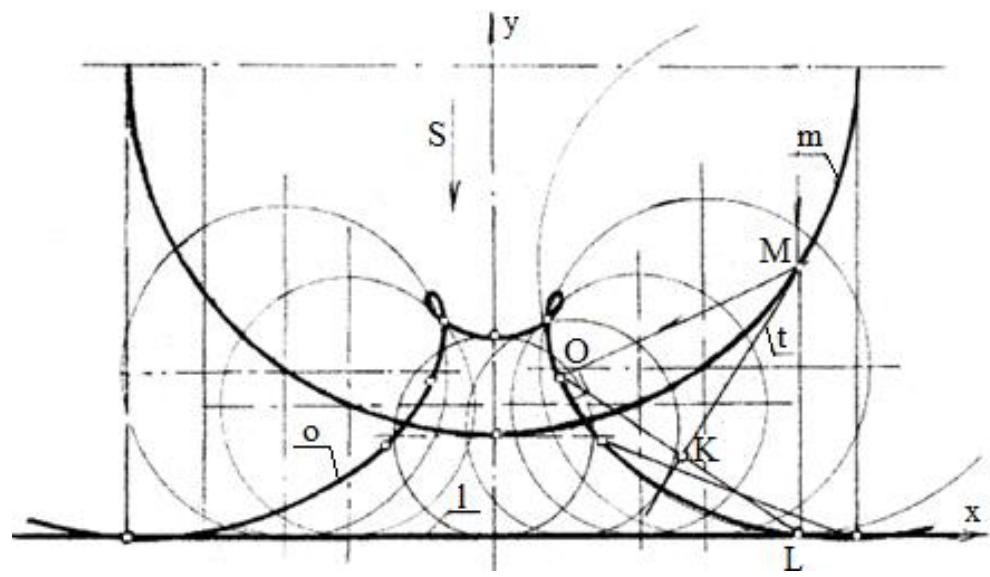

Fig. 6. Reflective curve is a circle and its orthotomics

Moreover, taking into account the direction of the incident rays perpendicular to the $\mathrm{x}$ axis, the $\mathrm{x}$-coordinate of the point $\mathrm{L}$ is equal to the $\mathrm{x}$-coordinate of the point $\mathrm{M}$ and the $\mathrm{y}$-coordinate of the point $\mathrm{M}$ is 0 , since the straight line 1 coincides with the $\mathrm{x}$-axis.

The point $\mathrm{K}$ of the intersection of the tangent $\mathrm{t}$ and the perpendicular to it is determined by the joint solution of equations (17) and (18)

$$
\begin{gathered}
X_{K}=\frac{x}{R^{2}}\left(\sqrt{R^{2}-x^{2}} \cdot y+R^{2}\right) \\
Y_{K}=\frac{y^{2}\left(R^{2}-x^{2}\right)}{R^{2}} .
\end{gathered}
$$

Figure 7 shows the result of the formation of four curves of the apparatus of reflections with a source at an infinity in which the reflecting curve is a circle.

The coordinates of the orthotomics point $\mathrm{O}(\mathrm{XoYo})$ are symmetric to the point $\mathrm{L}$ with respect to the tangent $\mathbf{t}$ are

$$
\begin{gathered}
X_{O}=2 X_{K}-X_{L} \\
Y_{0}=2 Y_{K}
\end{gathered}
$$


Caustics, Orthotomics, and Reflecting Curve with Source at an Infinity 9

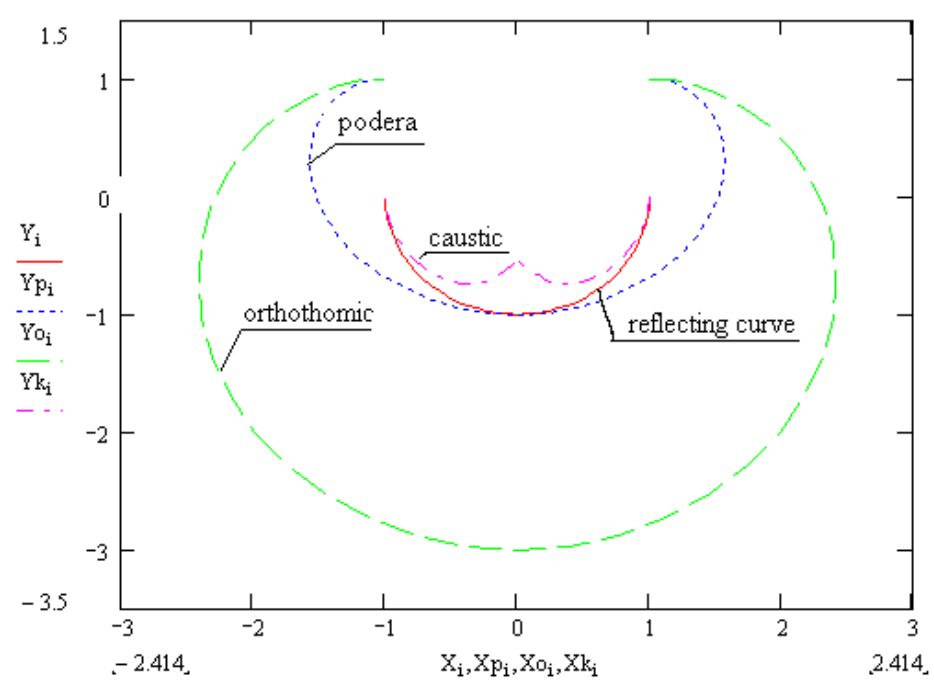

Fig. 7. The reflecting curve as a circle and its accompanying curves.

Substituting the coordinates of the points $\mathrm{K}$ and L into equations $(21,22)$, we obtain the coordinates of the point of orthotomics

$$
\begin{aligned}
X_{O} & =\frac{2 x y \sqrt{R^{2}-x^{2}}+x \cdot R^{2}}{R^{2}}, \\
Y_{O} & =\frac{2 y\left(R^{2}-x^{2}\right)}{R^{2}} .
\end{aligned}
$$

The $\mathrm{x}$ and $\mathrm{y}$ coordinates in equations (23) and (24) are the coordinates of a point on the reflective curve $\mathrm{m}$. In this example, the reflective curve is a circle.

\begin{tabular}{|c|c|c|c|}
\hline Reflecting & Podera & Orthothomic & Caustic \\
\hline $\begin{array}{l}x=R \cdot \cos \varphi \\
y=R \cdot \sin \varphi\end{array}$ & $\begin{array}{l}x_{p}=R \cdot \cos \varphi \cdot \\
\cdot\left(1-(\cos \varphi)^{2}\right)- \\
-b \cdot \sin \varphi \cdot \cos \varphi+x \\
y_{p}=b \cdot(\cos \varphi)^{2}+ \\
+R \cdot(\sin \varphi)^{3}\end{array}$ & $\begin{array}{l}x_{o}=-\sin 2 \varphi . \\
\cdot(b-R \cdot \sin \varphi)+ \\
+R \cdot \cos \varphi \\
y_{o}=b \cdot \cos 2 \varphi+ \\
+2 \cdot R \cdot(\sin \varphi)^{3}\end{array}$ & $\begin{array}{l}x_{k}=R \cdot \cos \varphi^{3} \\
y_{k}=R \cdot \sin \varphi . \\
\cdot\left(\frac{1}{2}+\cos \varphi^{2}\right)\end{array}$ \\
\hline
\end{tabular}

Table 3. Determining of reflecting apparatus curves on the given reflecting curve as a circle 
10 A. Dvoretskii.

\section{Conclusions}

The studied reflective properties of plane curves as sections of reflecting surfaces are preserved if these sections are generators of surfaces of revolution and rotative surfaces (the normal to the section at a given point coincides with the normal to the surface at the same point) and the source of the incident rays is in the same plane section.

The properties of the first reflection of curves can be investigated by their connections. It is giving us the possibility to construct new reflecting curves and reflecting systems with the demand properties. Caustics, orthotomics, and reflective curves with a source at infinity are a promising means of using the methods of geometric optics for modeling natural objects in ergo design.

\section{References}

1. Cayley A. A memoir upon caustics// Phil. Trans. Roy. Soc. Lond. 147:273-312; Collected Works. Vol. 2. pp. 336-380 (1857).

2. Glaeser G. Reflection on Spheres and Cylinders of Revolution. Journal for Geometry and Graphics// Volume 3. No. 2, p. 121 - 139 (1999).

3. Bruce J.W., Giblin P.J. Curves and Singularities: A Geometrical Introduction to Singularity Theory. Cambridge University Press. 262 p. (2012).

4. Bruce J.W., Giblin P.J., Gibson C.G. On caustics by reflection, Topology. I.21. 179 - 199 (1981).

5. Podgorny A.L., Dvoretsky A.T. Model of a plane reflective system for parallel incident rays// Proceedings of the Tavriya State Agrotechnical Academy. - Issue 4, volume 15.-Melitopol : TSAA, p.p. 12-15 (2002).

6. Dvoretsky A.T., Denisova T.V. Quasifocal lines// DOI: 10.30987/graphicon-2019-1-181184. 